\title{
Analysis of prostate bed motion using an endorectal balloon and cone beam computed tomography during postprostatectomy radiotherapy
}

This article was published in the following Dove Press journal:

OncoTargets and Therapy

24 May 2016

Number of times this article has been viewed

\section{Ji Hyeon Joo \\ Yeon Joo Kim \\ Young Seok Kim \\ Young Pil Cho \\ Ho Yeon Lee \\ Chang Young Jeong \\ Jungwon Kwak \\ Byung Chul Cho}

Department of Radiation Oncology, Asan Medical Center, University of Ulsan, College of Medicine, Seoul, Republic of Korea
Correspondence: Young Seok Kim Department of Radiation Oncology, Asan Medical Center, University of Ulsan, College of Medicine, 88 Olympic-ro 43 gil, Songpa-gu, Seoul 05505,

Republic of Korea

$\mathrm{Tel}+82230105614$

Fax +82230106950

Email ysk@amc.seoul.kr
Background: The authors conducted this prospective study to analyze the amount of interfractional prostate bed motion (PBM) and quantify its components with the use of an endorectal balloon (ERB).

Methods: A total of 1,348 cone beam computed tomography images from 46 patients who underwent postprostatectomy radiotherapy were analyzed. For the pilot image, electronic portal imaging, guided by skin marks was performed to ensure proper positioning and inflation of the ERB. Then, for bone matching, manual or automatic registration of the planning and each cone beam computed tomography was performed, based on the bony anatomy of the pelvis. Shifts (bony misalignment [BM]) in three directions were recorded at each treatment session. For prostate bed matching, manual matching was conducted based on the anterior rectal wall and the shift (PBM) was recorded. Total setup error was defined as the shift from the skin mark to the prostate bed matching, based on anterior rectal wall stretched by the ERB. PBM was defined as the difference between the total setup error and BM.

Results: Systematic errors for the total setup error were 1.0,1.3, and $1.0 \mathrm{~mm}$ in the right-left, anterior-posterior, and superior-inferior directions, with random errors of $1.9,2.4$, and $1.9 \mathrm{~mm}$, respectively. Systematic errors were 1.6, 1.6, and $0.3 \mathrm{~mm}$ for BM and 0.8, 1.1, and $0.9 \mathrm{~mm}$ for PBM, with random errors of 2.4, 2.5, and $1.1 \mathrm{~mm}$ for BM and 1.8, 2.2, and $1.9 \mathrm{~mm}$ for PBM.

Conclusion: The BM was the main component of the total setup error, suggesting that interfractional PBM was well controlled by the ERB device. Planning target volume margins of $<5 \mathrm{~mm}$ were needed to include $95 \%$ of the interfractional variations when using an ERB.

Keywords: prostate bed motion, endorectal balloon, cone beam computed tomography

\section{Introduction}

Biochemical relapse after radical prostatectomy can be controlled by radiotherapy (RT) to the prostate bed through two different schemes: salvage RT in patients with a postoperative prostate-specific antigen level $>0.2 \mathrm{ng} / \mathrm{mL}$, or adjuvant $\mathrm{RT}$ in patients with high risk factor(s), such as a pT3, or a positive surgical margin in the absence of any demonstrable disease. A dose-response relationship in the range of 60-70 Gy is suggested for both methods, ${ }^{1}$ particularly in the setting of salvage RT. ${ }^{2}$ However, rectal toxicity is the main factor limiting dose escalation, and the risk of grade $\geq 2$ toxicity or rectal bleeding is directly related to the volume of rectal tissue receiving a dose $\geq 60 \mathrm{~Gy}^{3}$

Three different strategies have been developed to overcome this obstacle: 1) intensity-modulated RT, which is used to reduce the irradiation dose delivered to the 
rectum; ${ }^{4} 2$ ) various image-guided RT techniques, which are employed to deliver the radiation as precisely as possible; ${ }^{5-7}$ and 3 ) devices, such as endorectal balloons (ERBs), ${ }^{8}$ rectal retractors, and spacers, which are used to reduce the movement of the rectum or to keep the posterior rectal wall away from the planning target volume (PTV). In particular, the ERB immobilizes the prostate gland by pushing it toward the pubic bone at a constant pressure. At the same time, the ERB retracts the posterior rectal wall away from the region being targeted by high-dose irradiation. In contrast to the definitive RT setting, the effect of an ERB on the immobilization of the prostate bed has not been validated prospectively in the postprostatectomy setting, except in retrospective analyses that compared two intensity-modulated RT protocols, either with or without an ERB. ${ }^{9,10}$ Therefore, the purpose of this study was to analyze the interfractional prostate bed motion (PBM) and quantify its individual components using an ERB and daily cone beam computed tomography (CBCT).

\section{Methods}

The CBCT datasets for 46 consecutive postprostatectomy patients who were treated with either salvage $(n=39)$ or adjuvant RT ( $\mathrm{n}=7)$ were included in this study. The patients were instructed to empty their bowels and bladder immediately before the planning computed tomography (CT) scan and every subsequent RT session. If residual stool or excessive gaseous distension was observed in the rectum on the prescan image, an enema was performed. Details regarding the planning CT and CBCT acquisition have been previously described. ${ }^{11}$ Briefly, the planning CT simulation was performed with the patient in the supine position and using an intravenous contrast agent. A knee-immobilization device was used, either with or without ankle immobilization (Knee-Lok and Foot-Lok positioned, CIVCO, Coralville, IA, USA). The region from the second lumbar vertebra to the proximal one-third of the femur was scanned with a Light Speed RT instrument (GE Healthcare, Little Chalfont, UK), with a slice thickness of $2.5 \mathrm{~mm}$. The CBCT images were acquired using the On-Board Imager System (OBI; Varian Medical Systems, Palo Alto, CA, USA), which was mounted on a linear accelerator, with a slice thickness of $2.5 \mathrm{~mm}$. The ERB was produced in-house. It comprised a $20 \mathrm{~cm}$ long flexible shaft made from rectal tubing with a rubber balloon attached at the end. The balloon was usually inflated with $60 \mathrm{cc}$ of air. The diameter and length of the inflated ERB were 40 and $60 \mathrm{~mm}$, respectively. After insertion and inflation, the ERB was fixed in place with an individual adjustable stopper.

The clinical target volume was defined according to the Radiation Therapy Oncology Group consensus guidelines. ${ }^{12}$ All patients received $66 \mathrm{~Gy}$ in 30 fractions delivered to the prostate bed. If obvious local relapse was evident from the imaging studies, an additional boost of 6.6 Gy (in three fractions) was delivered to the region. A three-step matching procedure was used. First, for the pilot image, and before each CBCT scan, electronic portal imaging (guided by skin marks) was performed to ensure proper positioning and inflation of the ERB. Second, for bone matching, manual or automatic registration of the planning $\mathrm{CT}$ and $\mathrm{CBCT}$ of each treatment session was performed based on the bony anatomy of the pelvis. Shifts (bony misalignment $[\mathrm{BM}]$ ) in the right-left (RL), anterior-posterior (AP), and superior-inferior (SI) directions were recorded at each treatment session. Third, for prostate bed matching, manual matching was conducted based on the anterior rectal wall and the shift (ie, the PBM) was recorded (Figure 1). The anterior rectal wall was considered a surrogate for the prostate bed because it is clearly identifiable and is one of the most vulnerable organs in the postprostatectomy RT setting.

The total setup error was defined as the shift from the skin marks to the prostate bed matching, based on the stretching of the anterior rectal wall by the ERB. The PBM was defined as the shift from the bony alignment to the prostate bed
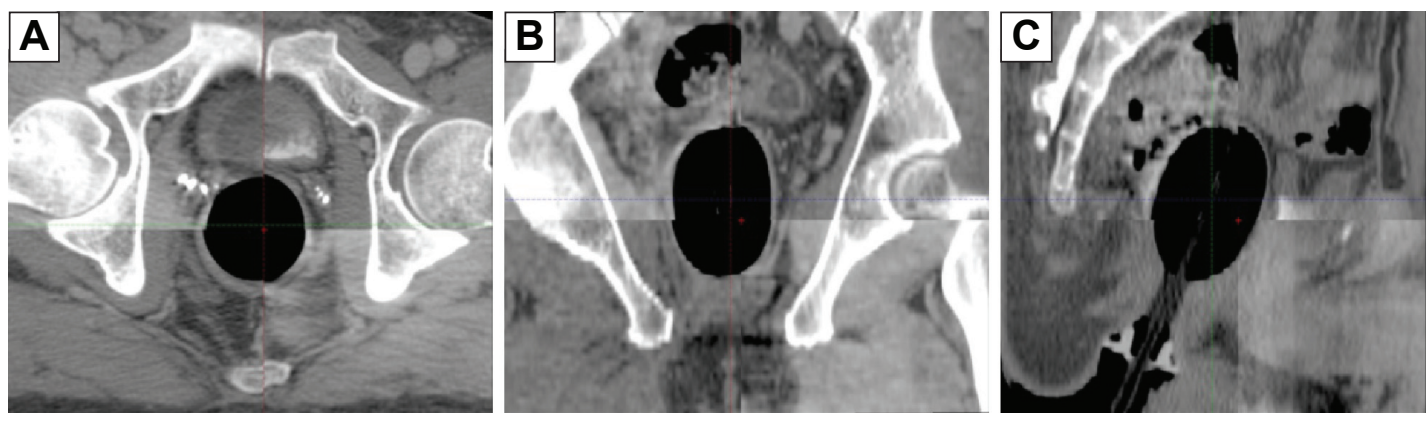

Figure I Example of the image guidance method using CBCT based on the anterior rectal wall: (A) axial, (B) coronal, and (C) sagittal image. Abbreviation: $\mathrm{CBCT}$, cone beam computed tomography. 
Table I Interfractional systematic and random variations (in $\mathrm{mm}$ ) for total setup error, BM, and PBM

\begin{tabular}{cllll}
\hline Variable & $\mathbf{M}$ & $\boldsymbol{\Sigma}$ & $\sigma$ & Margin $^{\mathbf{a}}$ \\
\hline Total setup error & & & & \\
RL & 0 & 1.0 & 1.9 & 5 \\
AP & 0.7 & 1.3 & 2.4 & 5 \\
SI & -0.7 & 1.0 & 1.9 & 5 \\
BM & & & & \\
RL & 0.5 & 1.6 & 2.4 & 5 \\
AP & 0.6 & 1.6 & 2.5 & 4 \\
SI & -0.1 & 0.3 & 1.1 & 4 \\
PBM & & & & \\
RL & -0.3 & 0.8 & 1.8 & 2 \\
AP & -0.3 & 1.1 & 2.2 & 4 \\
SI & 0.1 & 0.9 & 1.9 & 5 \\
\hline
\end{tabular}

Notes: Negative values indicate left, posterior, or inferior shift. aMargin to include $95 \%$ of interfractional variations.

Abbreviations: AP, anterior-posterior; BM, bony misalignment; $M$, group systematic deviations; PBM, prostate bed motion; RL, right-left; SI, superior-inferior; $\Sigma$, systematic deviation; $\sigma$, random deviation.

matching, or as the total setup error minus the BM. For the entire population, the global mean $(\mathrm{M})$ of each shifted value in each direction was calculated as the mean of all means. The distribution of systematic deviations $(\Sigma)$ was defined as the standard deviation of the mean deviation per patient. The random variation $(\sigma)$ was defined as the root-mean-square of the standard deviations of all patients. ${ }^{13}$ The random, systematic, and global mean of the variations, derived from the BM (denoted as $\sigma_{\mathrm{BM}}, \Sigma_{\mathrm{BM}}$, and $\mathrm{M}_{\mathrm{BM}}$, respectively) and the PBM (denoted as $\sigma_{\mathrm{PBM}}, \Sigma_{\mathrm{PBM}}$, and $\mathrm{M}_{\mathrm{PBM}}$, respectively) were calculated. The institutional review board from the Asan Medical Center deemed this study exempt from needing ethics approval as it corresponds to the review exemption conditions provided by the board, (Exemption confirmation number 2016-0493). Written informed consent was obtained from the patients.

\section{Results}

A total of 1,348 CBCT-based corrective shifts were performed and analyzed. The interfractional variations in the total setup error, BM, and PBM are summarized in Table 1. The interfractional displacement of the BM was larger than that of the PBM, except in the SI direction. This finding reflected the significant contribution of the BM to the total setup error.

For the total setup error, the percentage of shifts measuring $>5 \mathrm{~mm}$ was $3.3 \%, 3.6 \%$, and $3.0 \%$ in the RL, AP, and SI directions, respectively (Figure 2). The corresponding values were $3.0 \%, 1.1 \%$, and $0.6 \%$ for the $\mathrm{BM}$, and $0.4 \%$, $1.3 \%$, and $2.7 \%$ for the PBM in the RL, AP, and SI directions, respectively (Figures 3 and 4 ). The percentage of shifts that measured $\leq 2 \mathrm{~mm}$ were $64.8 \%, 69.6 \%$, and $75.3 \%$ in the RL, $\mathrm{AP}$, and SI directions for total setup error, respectively. The corresponding values were $67.8 \%, 79.1 \%$, and $82.9 \%$ for the $\mathrm{BM}$, and $96.7 \%, 79.2 \%$, and $80.6 \%$ for the PBM in the RL, $\mathrm{AP}$, and SI directions, respectively. Very little interfractional variation in the PBM was noted along the RL axis.

The whole procedure from ERB insertion to image registration and calculation of the corrective shift based on the prostate bed took $<15$ minutes. No complications were observed during RT, apart from mild discomfort on insertion of the ERB.

\section{Discussion}

The values for $\mathrm{M}, \Sigma$, and $\sigma$ for the PBM were smaller than the corresponding values for the BM in the RL and AP directions (Table 1). This finding is in line with those from other studies ${ }^{14,15}$ and indicated that the major part of the interfractional total setup error resulted from BM rather than from PBM. Thus, visualization of the pelvic bony anatomy using
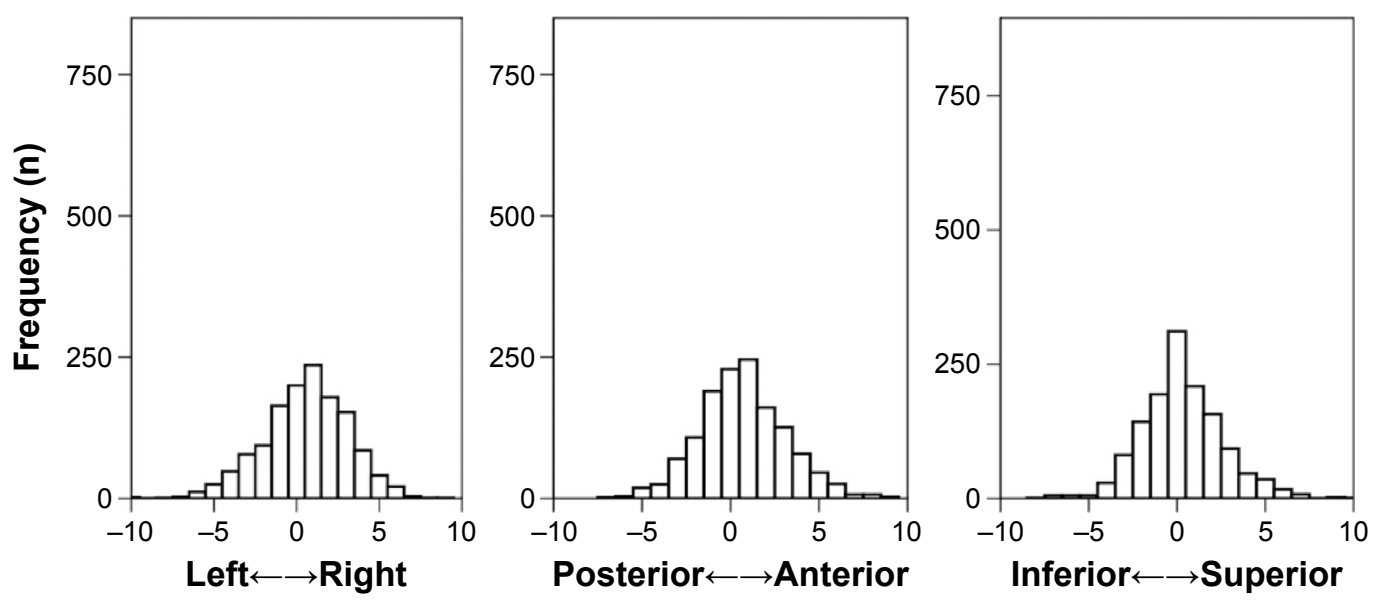

Figure 2 Frequency distributions of the total setup error in each direction (in $\mathrm{mm}$ ). 

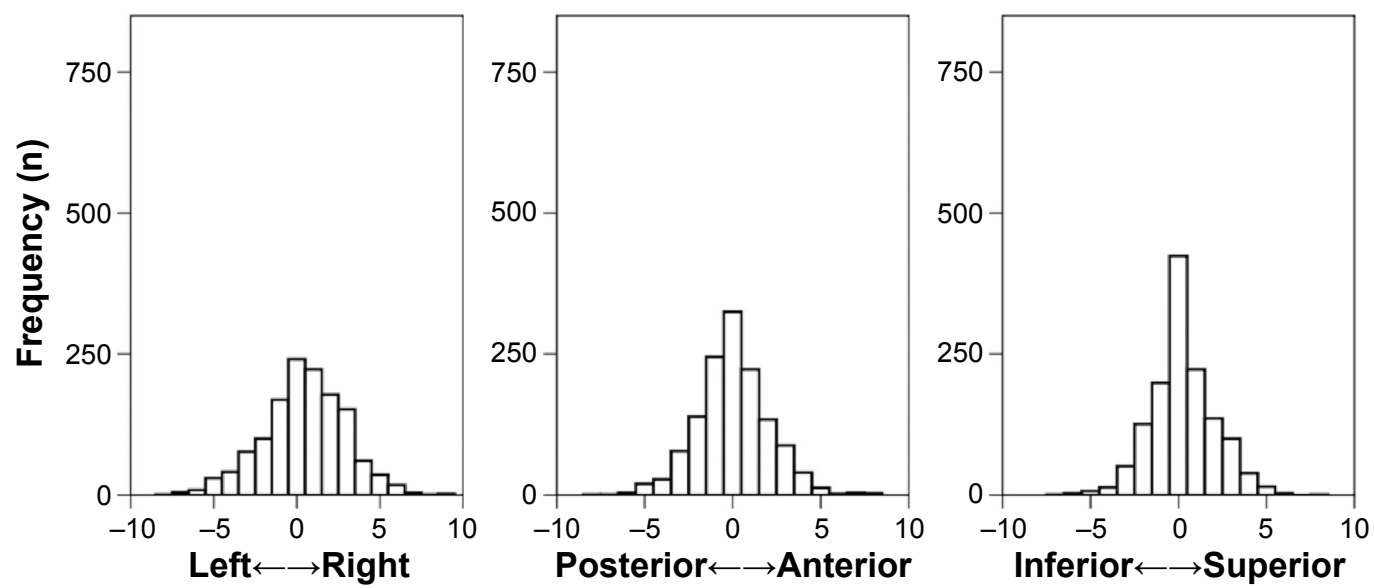

Figure 3 Frequency distributions of bony misalignment in each direction (in $\mathrm{mm}$ ).

an image-guided process, such as by $\mathrm{CBCT}$, or electronic portal images is important. After correcting for the BM, there was still a discrepancy between the bony alignment and the prostate bed matching, implying that both the pelvic bony anatomy and the anterior rectal wall should be directly visualized by CBCT. And daily CBCT is essential and cannot be substituted with the portal imaging based on bony anatomy only. The ERB fixed relatively well the prostate bed to the bony pelvis throughout the treatment. However, for SI movement, both $\Sigma_{\mathrm{PBM}}$ and $\sigma_{\mathrm{PBM}}$ were larger than $\Sigma_{\mathrm{BM}}$ and $\sigma_{\mathrm{BM}}$. The larger $\Sigma_{\mathrm{PBM}}$ and $\sigma_{\mathrm{PBM}}$ along the SI axis may be attributed to the variability in rectal content, which may affect the depth of ERB insertion. However, these differences were in the submillimeter range, a result that reflects the controllability of the PBM with an ERB.

The systematic and random components of the PBM were primarily in the AP direction, consistent with the findings of other studies in the postprostatectomy RT setting. ${ }^{16-18}$ The discrepancy in the systematic and random deviations between the AP and other directions was reduced by using an ERB (Table 2). Unlike other reports examining the interfractional PBM, the present study demonstrated that PBM was well controlled, particularly in the AP direction, when an ERB was used. The margins needed to include $95 \%$ of the variations in PBM were within 2, 4, and $5 \mathrm{~mm}$ in the $\mathrm{RL}$, AP, and SI directions, respectively. When only the interfractional PBM was considered, the results showed that $0.4 \%, 1.3 \%$, and $2.7 \%$ of each axis was outside the PTV, with an isotropic margin of $5 \mathrm{~mm}$. This result implied that less anisotropic margin expansion is needed. Intrafractional PBM, which was not addressed in the present study, should also be considered when deciding the PTV margin. To date, few studies have examined this issue, reporting an intrafractional PBM magnitude of $0.4 \mathrm{~mm}$, which is much smaller than the interfractional movement. ${ }^{16,19}$

Several investigators have studied the movement of an intact prostate using an ERB. Although one report did not show that the ERB helped to reduce interfractional prostate
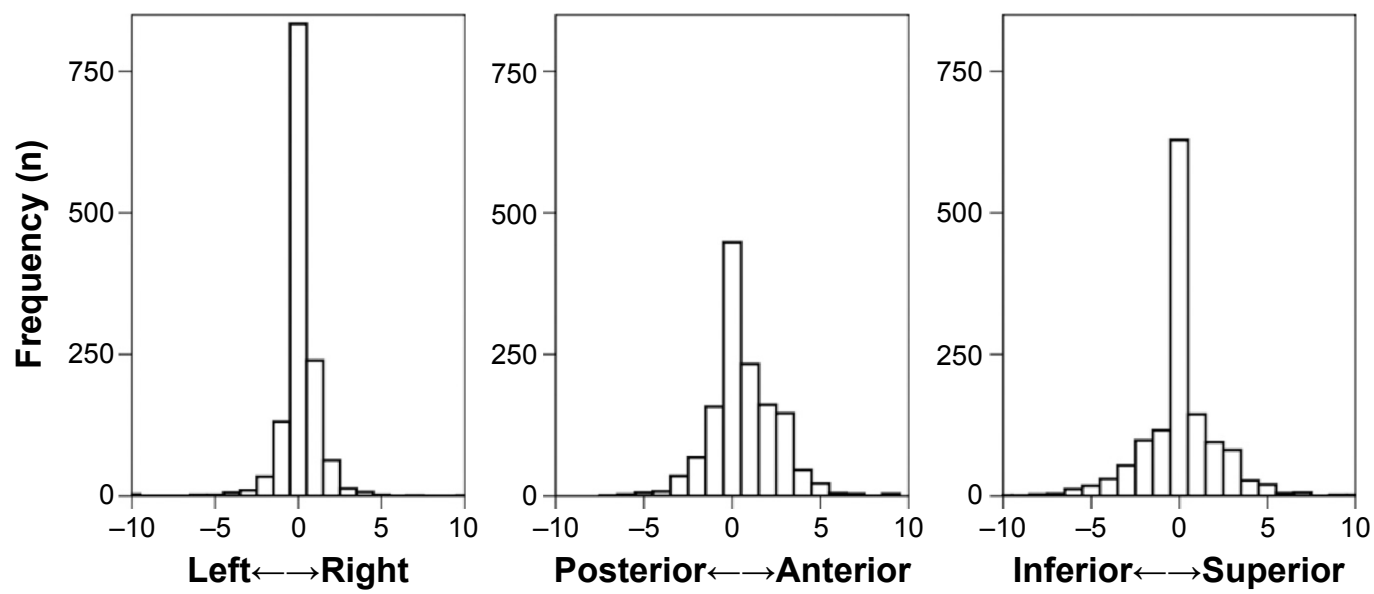

Figure 4 Frequency distributions of prostate bed motion in each direction (in $\mathrm{mm}$ ). 
Table 2 Literature review of interfractional PBM evaluated using CBCT

\begin{tabular}{|c|c|c|c|c|c|c|c|c|c|c|c|}
\hline \multirow[t]{2}{*}{ Study } & \multirow[t]{2}{*}{ СВСТ (n) } & \multirow[t]{2}{*}{ ERB } & \multicolumn{3}{|l|}{$M$} & \multicolumn{3}{|l|}{$\Sigma$} & \multicolumn{3}{|l|}{$\sigma$} \\
\hline & & & $\mathbf{R L}$ & $\mathbf{A P}$ & SI & $\mathbf{R L}$ & AP & SI & $\mathbf{R L}$ & AP & SI \\
\hline Kupelian et al ${ }^{17}$ & 140 & No & 0.1 & 0.4 & 0.1 & - & - & - & - & - & - \\
\hline Ost et $\mathrm{al}^{18}$ & 547 & No & 0 & 2.2 & 0.6 & 0.4 & 2.5 & 0.9 & 1.0 & 2.3 & I.4 \\
\hline Huang et $\mathrm{a}^{16}$ & 462 & No & 0 & 1.9 & -0.9 & 1.0 & 2.5 & 1.3 & 1.0 & 3.1 & 3.1 \\
\hline Present study & $\mathrm{I}, 348$ & Yes & -0.3 & -0.3 & 0.1 & 0.8 & 1.1 & 0.9 & 1.8 & 2.2 & 1.9 \\
\hline
\end{tabular}

Note: The dash indicates no data.

Abbreviations: AP, anterior-posterior; $\mathrm{CBCT}$, cone beam computed tomography; ERB, endorectal balloon; $M$, group systematic deviations; $n$, number; PBM, prostate bed motion; RL, right-left; SI, superior-inferior; $\Sigma$, systematic deviation; $\sigma$, random deviation.

motion, ${ }^{8}$ ERBs are still widely used to immobilize the prostate gland ${ }^{20}$ and reduce the RT dose delivered to the rectal wall. ${ }^{21}$ Even when using an ERB, it is important to maintain consistent rectal and bladder volumes throughout RT. ${ }^{8}$ In the present study, the rectal and bladder volumes were directly visualized by $\mathrm{CBCT}$ on a daily basis, and the patients were instructed to empty their bowels and bladder before each treatment. Such methods helped to minimize the PBM, especially in the AP direction. In addition to variations in rectal content, Fiorino et $\mathrm{al}^{22}$ reported variations in bladder volume in postprostatectomy RT patients. In the current study, the patients had empty bladders before every treatment session. In practice, the patients tend to complain of urgency and frequency during RT; therefore, it is not always easy for patients to fill their bladder as constantly as possible. Although bladder content and movement cause variations in PBM, it is the rectal content and movement that are primarily responsible. After prostatectomy, the bladder neck is partly fixed to the bony anatomy by postoperative fibrotic tissue. Therefore, filling the bladder only reduces its movement to a small extent.

Although we did not make a direct comparison between the interfractional movement of the prostate bed with and without ERB in the present study, we enrolled the patients prospectively according to standard guidelines. To the best of our knowledge, this is the first study to report interfractional PBM during RT using an ERB and daily CBCT in such a large cohort.

\section{Conclusion}

The present study demonstrated that the interfractional PBM was small, with group systematic deviations of $\leq 0.3 \mathrm{~mm}$ along all the three axes. As shown in the current study, a margin of $<5 \mathrm{~mm}$ was required to include $95 \%$ of the interfractional variations to calculate the total setup error with the use of ERB and daily CBCT. The use of ERB might be recommended to limit interfractional $\mathrm{PBM}$ in patients who underwent postprostatectomy RT unless they complain of moderate-to-severe discomfort on ERB insertion. Comparative study between ERB with non-ERB group is warranted.

\section{Acknowledgments}

This study was supported by a grant (2013-0160) from the Asan Institute for Life Science, Seoul, Korea. This study was presented in Radiological Society of North America annual 2014 meeting as a poster.

\section{Disclosure}

The authors report no conflict of interest in this work.

\section{References}

1. King CR, Kapp DS. Radiotherapy after prostatectomy: is the evidence for dose escalation out there? Int J Radiat Oncol Biol Phys. 2008;71(2): 346-350.

2. King CR, Spiotto MT. Improved outcomes with higher doses for salvage radiotherapy after prostatectomy. Int J Radiat Oncol Biol Phys. 2008; 71(1):23-27.

3. Michalski JM, Gay H, Jackson A, Tucker SL, Deasy JO. Radiation dose-volume effects in radiation-induced rectal injury. Int $J$ Radiat Oncol Biol Phys. 2010;76(3 Suppl):S123-S129.

4. Staffurth J, Radiotherapy Development Board. A review of the clinical evidence for intensity-modulated radiotherapy. Clin Oncol (R Coll Radiol). 2010;22(8):643-657.

5. Langen KM, Zhang Y, Andrews RD, et al. Initial experience with megavoltage (MV) CT guidance for daily prostate alignments. Int $J$ Radiat Oncol Biol Phys. 2005;62(5):1517-1524.

6. Boda-Heggemann J, Lohr F, Wenz F, Flentje M, Guckenberger M. kV cone-beam CT-based IGRT: a clinical review. Strahlenther Onkol. 2011;187(5):284-291

7. Pinkawa M, Pursch-Lee M, Asadpour B, et al. Image-guided radiotherapy for prostate cancer. Implementation of ultrasound-based prostate localization for the analysis of inter- and intrafraction organ motion. Strahlenther Onkol. 2008;184(12):679-685.

8. van Lin EN, van der Vight LP, Witjes JA, Huisman HJ, Leer JW, Visser AG The effect of an endorectal balloon and off-line correction on the interfraction systematic and random prostate position variations: a comparative study. Int J Radiat Oncol Biol Phys. 2005;61(1):278-288.

9. Jameson MG, De Leon J, Windsor AA, et al. Endorectal balloons in the post prostatectomy setting: do gains in stability lead to more predictable dosimetry? Radiother Oncol. 2013;109(3):493-497.

10. Smeenk RJ, van Lin EN, van Kollenburg P, McColl GM, Kunze-Busch M, Kaanders JH. Endorectal balloon reduces anorectal doses in post-prostatectomy intensity-modulated radiotherapy. Radiother Oncol. 2011; 101(3):465-470.

11. Choi HJ, Kim YS, Lee SH, et al. Inter- and intra-observer variability in contouring of the prostate gland on planning computed tomography and cone beam computed tomography. Acta Oncol. 2011;50(4):539-546. 
12. Michalski JM, Lawton C, El Naqa I, et al. Development of RTOG consensus guidelines for the definition of the clinical target volume for postoperative conformal radiation therapy for prostate cancer. Int J Radiat Oncol Biol Phys. 2010;76(2):361-368.

13. van Herk M. Errors and margins in radiotherapy. Semin Radiat Oncol. 2004;14(1):52-64.

14. Nairz O, Merz F, Deutschmann H, et al. A strategy for the use of imageguided radiotherapy (IGRT) on linear accelerators and its impact on treatment margins for prostate cancer patients. Strahlenther Onkol. 2008; 184(12):663-667.

15. Zucca S, Carau B, Solla I, et al. Prostate image-guided radiotherapy by megavolt cone-beam CT. Strahlenther Onkol. 2011;187(8):473-478.

16. Huang K, Palma DA, Scott D, et al. Inter- and intrafraction uncertainty in prostate bed image-guided radiotherapy. Int J Radiat Oncol Biol Phys. 2012;84(2):402-407.

17. Kupelian PA, Langen KM, Willoughby TR, Wagner TH, Zeidan OA, Meeks SL. Daily variations in the position of the prostate bed in patients with prostate cancer receiving postoperative external beam radiation therapy. Int J Radiat Oncol Biol Phys. 2006;66(2):593-596.
18. Ost P, De Meerleer G, De Gersem W, Impens A, De Neve W. Analysis of prostate bed motion using daily cone-beam computed tomography during postprostatectomy radiotherapy. Int J Radiat Oncol Biol Phys. 2011;79(1):188-194.

19. Zhu M, Bharat S, Michalski JM, Gay HA, Hou WH, Parikh PJ. Adaptive radiation therapy for postprostatectomy patients using real-time electromagnetic target motion tracking during external beam radiation therapy. Int J Radiat Oncol Biol Phys. 2013;85(4):1038-1044.

20. D'Amico AV, Manola J, Loffredo M, et al. A practical method to achieve prostate gland immobilization and target verification for daily treatment. Int J Radiat Oncol Biol Phys. 2001;51(5):1431-1436.

21. Patel RR, Orton N, Tome WA, Chappell R, Ritter MA. Rectal dose sparing with a balloon catheter and ultrasound localization in conformal radiation therapy for prostate cancer. Radiother Oncol. 2003; 67(3):285-294.

22. Fiorino C, Foppiano F, Franzone P, et al. Rectal and bladder motion during conformal radiotherapy after radical prostatectomy. Radiother Oncol. 2005;74(2):187-195.
OncoTargets and Therapy

\section{Publish your work in this journal}

OncoTargets and Therapy is an international, peer-reviewed, open access journal focusing on the pathological basis of all cancers, potential targets for therapy and treatment protocols employed to improve the management of cancer patients. The journal also focuses on the impact of management programs and new therapeutic agents and protocols on

\section{Dovepress}

patient perspectives such as quality of life, adherence and satisfaction. The manuscript management system is completely online and includes a very quick and fair peer-review system, which is all easy to use. Visit http://www.dovepress.com/testimonials.php to read real quotes from published authors. 